\title{
Controlling Magnetic Flux Density in Active Magnetic Bearings
}

\author{
Prince Owusu-Ansah ${ }^{1}$, Alex Frimpong Justice ${ }^{2}$, Timothy Alhassan ${ }^{3}$, \\ Saviour Kwame Woangbah ${ }^{4}$. \\ ${ }^{1}$ School of Mechanical and Electronic Engineering, Wuhan University of Technology \\ ${ }^{1}$ Mechanical Engineering Department, Kumasi Polytechnic P. O. Box 854, Kumasi Ghana \\ ${ }^{2,3,4}$ Mechanical Engineering Department, Kumasi Polytechnic P. O. Box 854, Kumasi Ghana
}

\begin{abstract}
This paper discusses the control of magnetic flux density in active magnetic bearings. The rotor is laminated in which the magnetically active part of the rotor is built with stacks of disk consisting of shaped layers of ferromagnetic sheets which are electrically insulated from each other.

The use of different applications of control measures depending on its application, linearity, measuring range, resolution, sensitivity as well as its frequency range causes the output signal of its sensors to change according to its physical effect as a function of the measured quantity during the process of measurements.

Finally, different types of displacement sensor, it's measuring principles and the various types of application used in the measurement of magnetic flux density has been studied and presented.
\end{abstract}

Keywords: Active magnetic bearing, Current Measurement, Magnetic flux density, Rotor, Sensor,

\section{Introduction}

Magnetic bearings are bearings which supports loads using magnetic levitation [1], they support moving machinery without making physical contact with each other, for example, they can levitate a rotating shaft and permit relative motion without friction or wear, they are essentially used in industrial applications such as electric power generation, petroleum refining, machine tool operation, natural gas pipelines, zippe-type etc.

Active magnetic bearings (AMB) are clearly favored over passive bearings [2], because they are actively controlled by means of electromagnet and results in high power loss due to the presence of a biased current, but the inclusion of suitable feedback control loops and other elements such as sensors and power amplifier makes its more preferable than passive bearing due to its lack of active control damping capabilities $[3,4]$ Passive magnetic bearing consists of permanent magnetic placed in a position that can levitate an object in this case, the rotor making it contact free using the principles of magnetic effect $[5,6]$.

The first group of magnetic bearing classifications are referred to as reluctance force bearings, whiles the second group is made up by the Lorentz force bearings. Whereas the latter type of bearings has lately gained an increasing importance mainly in the field of the self-bearing motors, it is still the case that the bulk of industrial magnetic bearing applications employ reluctance force bearings. In such bearing designs magnetic flux provides a bias flux which flows radially in an open iron-core ring and then moves directly into the four poles of the stator through the airgaps and then moves through the stator alongside the axial direction before it finally returns to the rotor via the other airgaps.

The electromagnet flux generated from the upper and lower magnets coils passes down through the rotor along the radial direction on one side, and then passes up through the other side of the rotor finally. Thus, if the total flux increases in the lower side, it then results in the reduction of magnet flux at the upper portion of the rotor, the resultant difference of the magnetic flux as a result of this process produces a control force in the magnetic bearing for the suspension and levitation action for the rotor to be suspended and control very effectively throughout it rotational operation.

\section{Magnetic Bearing As A Controlled Suspension}

Active magnetic bearing consists of a copper coil, in some cases high temperature superconductors which provides high magnetic flux ensuring contact free movement in these designs [7].

AMB has good performance and with a microprocessor control based it compensates the instability that will occur in the system [8]. In active magnetic it is possible to design and adapt different functionalities in relation to different points of view of which the most important are of the following.

Position of the active circuit: The position of the active circuit in the design is done keeping in mind the main function of $\mathrm{AMB}$ are to levitate, sustain and align the rotating mass of the flywheel rotor. The active bearing structure has a coil placed inside it which constitutes the active circuit of the AMB, that suppliers the coil current to the system. 


\section{Relation Between Current And Force In An Active Magnetic Bearing}

It is assumed that the magnetic flux $\phi\left[T . \mathrm{m}^{2}\right]$, in the stator cross-section $\mathrm{A}_{\mathrm{fe}}\left[\mathrm{m}^{2}\right]$ is constant along the whole loop. $A_{f e}=A_{a}\left(A_{a}\right.$ is the cross-section of the air-gap), Thus,

$$
\begin{aligned}
& \phi=\mathrm{B}_{\mathrm{fe}} \mathrm{A}_{\mathrm{fe}}=\mathrm{B}_{\mathrm{a}} \mathrm{A}_{\mathrm{a}} \\
& \mathrm{B}_{\mathrm{fe}}=\mathrm{B}_{\mathrm{a}}=\mathrm{B}
\end{aligned}
$$

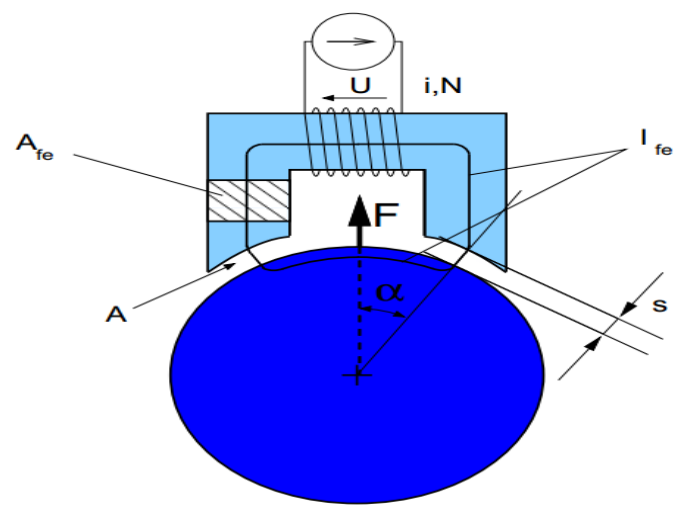

Fig. 1. Magnetic force of an AMB.

The magnetic path is decomposed two parts, the field in the air, and in the soft magnetic material

$$
\begin{aligned}
& \mathrm{B}=\mu \mu_{\mathrm{o}} \mathrm{H} \\
& \mathrm{N}_{\mathrm{i}}=\mathrm{H}_{\mathrm{fe}} \mathrm{l}_{\mathrm{fe}}+2 \mathrm{H}_{\mathrm{a}} \mathrm{s}
\end{aligned}
$$

Where $\mathrm{s}[\mathrm{m}]$ is the air gap between stator and rotor. The average magnetic path length in the lamination is $1_{\mathrm{fe}}$ $[\mathrm{m}]$.

From Eq. (3) and Eq. (4) and solving for B yields Equation. (5) as the flux density.

$\mathrm{B}=\mu_{\mathrm{o}} \mathrm{Ni} /\left(\left(\mathrm{l}_{\mathrm{fe}} / \mu_{\mathrm{r}}\right)+2 \mathrm{~s}\right)$

Since the iron relative permeability $\mu_{\mathrm{r}} » 1$, the magnetization of the iron can often be neglected, and the relation can be written as

$$
\mathrm{B} \approx \mu_{\mathrm{o}} \mathrm{Ni} / 2 \mathrm{~s}
$$

The AMB force is obtained by considering the energy stored in the air gap and is given by equation. (7)

$$
\mathrm{F}=\mathrm{B}_{\mathrm{a}}^{2} \mathrm{~A}_{\mathrm{a}} / \mu_{\mathrm{o}}
$$

The angle between the force direction and the center of the cross-section A is determined by $\alpha$. In a four poles radial AMBs, which means eight actuator teeth, $\alpha$ is $22.5^{\circ}$. Equation (6) and equation (7) results in the force for one actuator:

$$
\begin{aligned}
& \mathrm{F}=1 / 4 \mu_{0} \mathrm{~N}^{2} \mathrm{~A}_{\mathrm{a}}\left(\mathrm{i}^{2} / \mathrm{s}^{2}\right) \cos \alpha=\mathrm{k}\left(\mathrm{i}^{2} / \mathrm{s}^{2}\right) \cos \alpha \\
& \mathrm{F}=1 / 4 \mu_{0} \mathrm{~N}^{2} \mathrm{~A}_{\mathrm{a}}
\end{aligned}
$$

In order to produce a magnetic force along two opposite directions, the actuators are arranged in pairs as indicated in fig. 1 this enables a full control of the rotor along one axis. For the pair of magnets, the force $F_{x}$ $[\mathrm{N}]$ represents the force difference between the positive and the negative directions, the actuator currents are defined as the sum of a bias current $i_{0}[A]$ and a control current $i_{x}[A]$ for the positive actuator, and the difference $\left(i_{0}-i_{x}\right)$ for the negative actuator. The air gaps are defined by the deviation $x[\mathrm{~m}]$ and the nominal air gap $s_{o}[\mathrm{~m}]$, thus the terms $\left(\mathrm{s}_{\mathrm{o}}-\mathrm{x}\right)$ and $\left(\mathrm{s}_{\mathrm{o}}+\mathrm{x}\right)$ are inserted.

$$
\begin{aligned}
& \left.F_{x}=F_{+}-F_{-}=k\left(\left(i_{o}+i_{x}\right)^{2} /\left(s_{0}-x\right)^{2}\right)-\left(i_{o}-i_{x}\right)^{2} /\left(s_{o}+x\right)^{2}\right) \cos \alpha \\
& k=1 / 4 \mu_{0} N^{2} A_{a} \\
& \text { By considering that }\left(x \ll s_{o}\right) \text { and }\left(i_{x} \ll i_{o}\right) \text {, equation (10) can be linearized using Taylor's series. It yields }
\end{aligned}
$$
the typical AMB relation in Equation. (13).

$$
\begin{aligned}
& \mathrm{F}_{\mathrm{x}}=4 \mathrm{k}\left(\mathrm{i}_{\mathrm{o}} / \mathrm{s}_{\mathrm{o}}{ }^{2}\right)(\cos \alpha) \mathrm{i}_{\mathrm{x}}+4 \mathrm{k}\left(\mathrm{i}_{\mathrm{o}} / \mathrm{s}_{\mathrm{o}}{ }^{2}\right)(\cos \alpha) \mathrm{x}=\mathrm{k}_{\mathrm{i}} \mathrm{i}_{\mathrm{x}}+\mathrm{k}_{\mathrm{x}} \mathrm{x} \\
& \mathrm{K}_{\mathrm{s}}=\mathrm{k}_{\mathrm{i}}\left(\mathrm{i}_{\mathrm{o}} / \mathrm{s}_{\mathrm{o}}\right)
\end{aligned}
$$

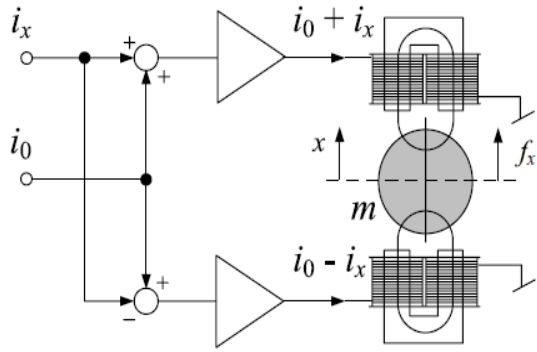

Fig. 2. Differential bearing mode of the bearing magnets. 
The coefficient $\mathrm{k}_{\mathrm{s}}$ is the open-loop stiffness in which $\mathrm{i}$ is the inherent negative stiffness. This negative stiffness leads to open-loop instability. The force-current coefficient $\mathrm{k}_{\mathrm{i}}$ is the actuator gain.

\section{Structural Configuration Of Radial Electromagnet Bearing}

In the structural configuration of the radial electromagnet distinctions are made on the basis of their electromagnetic polarities whiles the rotor rotates. When the magnetic flux is confined to a plane perpendicular to the axis of the rotation the magnetic pole is made to alternate in a polarity around the rotor as indicated in Fig. 3a. Similarly, if portions of the magnetic flux are made to pass axially along the rotor or the stator as shown in Fig. 3b, the arrangements ensures that all the poles in that given plane attains the same polarity thereby allowing alternation of the polarity to occur in the axial direction. There is always polarity alternation within the system no matter the configuration which ensures that the total magnetic flux passing through the surface of the rotor is always equal to zero. In order to ensure that the eddy current loss are kept as low as possible, the rotor is laminated in which the magnetically active part of the rotor is built with a stack of disk consisting of shaped layers of ferromagnetic sheets which are electrically insulated from each other.

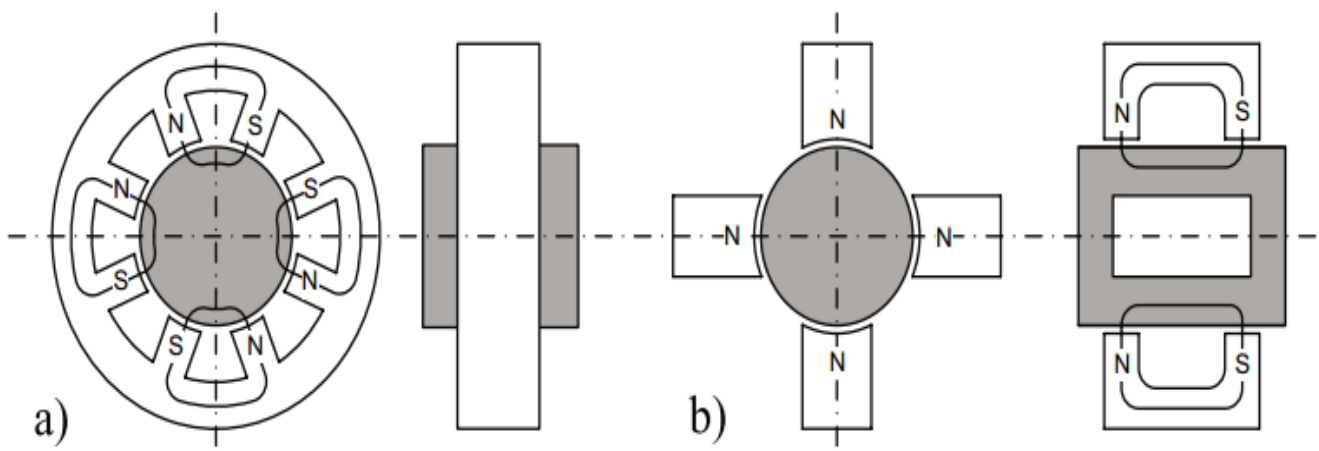

Fig. 3. (a) Field lines perpendicular to the rotor axis b) Field line parallel to the rotor axis.

The flux path shows that flux permanent magnet bias flux flows into the rear side stator pole then the flux flows into the rotor via air gap. Afterwards, the flux returns from rotor to the permanent magnet via the far side rotor, at conditions when no static load or any external disturbances are experienced by the rotor, no current flows through the coil and is only the bias fluxes which are made to flow through the air gaps by permanent magnet, as such the air gap flux densities are almost identical and equal in nature.

The magnetic flux density in difference will act force on the rotor, the rotor at the center position sum of the force must be zero.

\section{Load Capacity Of A Magnetic Bearing}

The load capacity of a magnetic bearing is the force obtained with the maximum admissible magneto motive force $N I_{\max }$.

The achievable magneto motive force $N I_{\max }$, i.e. the product of the maximum current $i_{\max }$ and winding number $n$, depends on the available winding cross section, the mean winding length, and the achievable heat dissipation. The maximum heat dissipation depends on the kind and amount of cooling. For an effective computation of the load capacity it is therefore essential to first calculate the relevant cooling capability. In the bearing magnet both the iron of the magnetic loop and the copper of the winding require space.

In order to optimize the bearing geometry, we distribute the space available in the bearing magnet optimally between iron and copper for maximum load capacity. Optimization can already be achieved with a simple model of the magnetic loop. However, this model can still be refined by considering, for instance, the stray flux effect, as long as it can be easily modeled, or by admitting different cross sections in the iron and the air gap, as they occur with magnets with pole shoes. Finally, the load capacity of the optimized bearing geometry can be checked by finite element modeling.

Whether the magnetic flux is generated with high current and a low number of windings or else with low current and a great number of windings is irrelevant for the optimization of the bearing geometry. Variations in the coil design allow matching the magnetic bearing with the power amplifier.

\section{Sensors}

An important aspect for the effective performance of high speed industrial machinery which used magnetic bearing as a means of levitating the high speed rotor depend basically on the characteristic of the its displacement sensors used. In order to measure the position of the moving rotor contact free sensors must be 
used which will be able to measure on a rotating surface. Consequently. The geometry of the rotor, i.e. its surface quality, and the homogeneity of the material at the sensor will also be an influencing factor measuring and obtaining its results. A bad surface will thus produce noise disturbances, and consequently geometry errors will eventually cause disturbances with the rotational frequency of the rotor or with its multiples thereof.

\section{Displacement Measurement}

Selection of a displacement sensors should depend on the type of magnetic bearing, the linearity, its measuring range, resolution, sensitivity as well as its frequency range must all be well taken into accounts.

- Temperature range, temperature drift of the zero point and its sensitivity

- Electrical factors such as grounding issues associated with capacitive sensors

- Mechanical factors such as shock and vibration

- Environmental factors such as dust, aggressive media. Vacuum, or radiation

- Noise immunity against other sensors, magnetic alternating fields of the electromagnets, electromagnetic disturbances from switch amplifiers

\section{Inductive Displacement Sensors}

Ferromagnetic cores of an inductor coil are driven by an oscillator as shown in Fig. 4a. In the situation when the ferromagnetic object whose position needs to be measured, approaches the coil its inductance changes. The change in inductance is sensed by an electronics and is converted into a sensor output voltage which is proportional to the displacement. Two sensors which opposes each other are frequently arranged on a rotor as indicated in Fig. 4b which are operated differentially in a bridge circuit which consist of a constant bridge frequency which produces a nearly linear signal. Modulation frequency of inductive sensors varies from $5 \mathrm{~Hz}$ up to $100 \mathrm{~Hz}$. The cut- off frequency of the output signal lies in a range between one tenth and one fifth of the modulation frequency.

Inductive sensors are not overly sensitive to external magnetic fields near bearing magnets as long as the fields which are not sufficient to modulate the permeability of the sensor core material. Moreover, massive disturbance usually occurs when the magnetic bearings are driven by switched power amplifiers in which the switching frequency of the amplifier is closer to the modulation frequency.

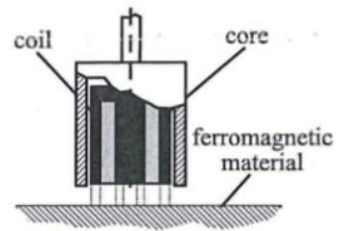

(a) Sensor head

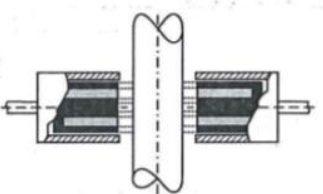

(b) Differentially measuring sensors

Fig. 4. Inductive displacement sensor

\section{Eddy Current Sensors}

The electromagnetic coil is made to induce eddy currents in a conductive object to be measured, hence absorbing energy from the oscillating circuit. High-frequency alternating is made to run through the air-coil which is embedded in the housing. Depending on the clearance to be measured, the inductance of the coil varies, and external electronic circuitry converts this variation into an output signal. The accepted modulation frequencies ranges between $1-2 \mathrm{MHz}$, resulting in a useful measuring frequency ranges of $0 \mathrm{~Hz}$ up to approximately $20 \mathrm{kHz}$.

The use of sensor may usually cause some mutual interference, as such the minimum clearance that exist between sensors is usually defined in the bearing mounting guide. A minimum defined clearance must be adhered to when operating several sensors surrounding conducting material. In operating several sensors in the same system, the modulation frequencies must be synchronized, but however synchronization may not be possible in all sensors systems.

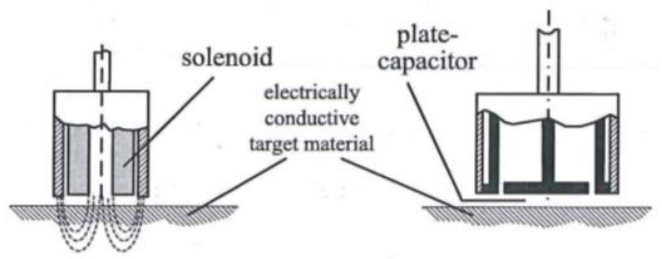

Fig. 5. (a) Eddy -current displacement sensor (b) Capacitive sensor 


\section{Eddy Current Radial Displacement Sensor ON A PCB Transverse Flux Sensor}

To minimize space requirement and for the purpose to save production cost of the sensor it is usually desirable to place the eddy current sensor coils directly on a printed circuit board (PCB) which is placed around the rotor, such a design is called Transverse Flux Sensor [9].

The magnetic field of the coil in an eddy current displacement sensor is normally directed towards the rotor in such a way that the axis of the rotor of the radial sensor coil is perpendicular to its axis of rotation in Fig. 6.

This arrangement is made to provide a suitably sensitivity to a target displacement only is specific direction which is perpendicular to the coil surface, where sensitivity to displacements in any lateral direction is poor. This behavior is due to the fact that magnetic field strength is very high at the coil's center and that its maximum field strength is very high at the coil center and that the maximum field gradient component which is perpendicular to its surface.
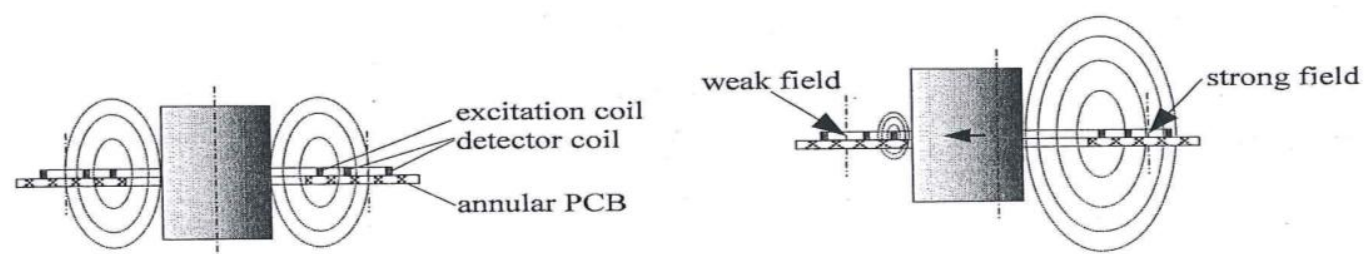

(a) Rotor concentric within the sensor (b) With rotor displacement

Fig. 6. Transverse Flux Sensor

The strength of the electro-magnetic field of the excitation is dependent on the position of the rotor within the excitation coil measuring this field strength with the detection coil will give the high lateral sensitivity in the $\mathrm{x}$ and $\mathrm{y}$ directions.

\section{Capacitive Displacement}

The capacity of a plate capacitor varies with its clearance. In capacitive measuring method, the sensor and the opposing object which is to be measured forms one electrode of the plate. Fig. 5b. In the measuring system, an alternating current which constant of a constant frequency runs through the sensor. The voltage of the sensor is proportional to the clearance between the sensor electrode and the object which is to be measured, and it is demodulated and amplifier by a special circuit. The bandth width of the output signal ranges between $5 \mathrm{kHz}$ and $100 \mathrm{kHz}$

The electrostatic charging of the countless rotor will cause interferences. The sensors are sensitive to dirt which could modify the dielectric constant in the air gap.

\section{Magnetic Displacement Sensors}

When current $i$ in a magnetic displacement sensor is kept constant in a magnetic loop within an air gap, the flux density $B$ can be used to measure the size of the air gap. In Fig. 7, a well linearized displacement signal results from the difference between the measured flux density $U_{B D}-U_{B n}$. Flux density $B$ is measured with a Hall sensor or with a field plate. Magnetic displacement sensors are very sensitive to interference by external fields.

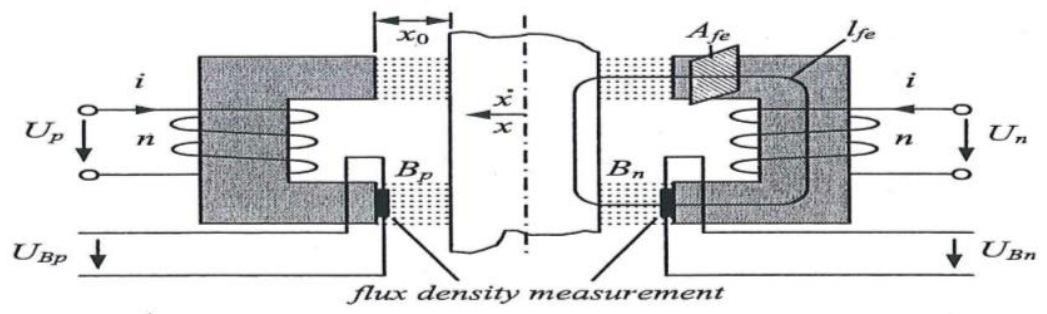

Fig. 7. Combined displacement -velocity sensor

\section{Velocity Measurement}

When the current $i$ is kept constant in a magnetic circuit, the flux $\Phi$ varies with the air gap. The voltage $U$ is proportional to the derivative $\mathrm{d} \Phi / \mathrm{dt}$ and the velocity $\mathrm{dx} / \mathrm{dt}$ When the combined displacement- velocity sensor as shown in Fig. 7, is used, the difference between the voltages $U_{p}-U_{n}$ yields a nearly velocity signal. This type of sensor is very appropriate for measuring displacement and velocity; further permanent magnet can also be used in this situation instead of electric excitement. 


\section{Flux And Current Measurement \\ Hall Effect}

In situations where current travels along a piece of thin, a band- shaped conductor, and when this conductor lies in a magnetic field which is perpendicular to the band plane, forces which are made to act perpendicular to their band on the electrons moves at a drifting speed $v$ along the conductor as indicated in Fig 8. This usually leads to accumulation of positive and negative charges on both longitudinal sided within the band, and consequently to an electric voltage $U_{b}$. This Hall voltage is proportional to the flux density $B$ and the current $i[10]$.

$$
U_{b}=k_{h} B i
$$

The proportionality factor $k_{h}$ depends on the type of geometry of the conductor and its material, in measuring the flux density, the Hall sensor is driven by a constant - current source. Hall sensors can be optimized regarding the size of the proportionality factor $k_{h}$ and the temperature drift of the zero point.

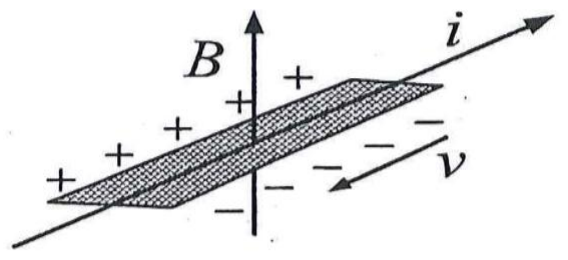

Fig. 8. Hall Effect

\section{Coil And Integrator}

The law of induction states that the voltage across a coil with $\mathrm{n}$ turns is given as $\mathrm{U}=\mathrm{nd} \Phi / \mathrm{dt}$

When a measurement coil is mounted on a magnet the resulting voltage is fed to an electronic integrator, the integrator output signal will be proportional to the magnetic flux through the measurement coil as shown in Fig. 9 This method, however, has the disadvantage that only the alternating components of the flux can therefore be measured.

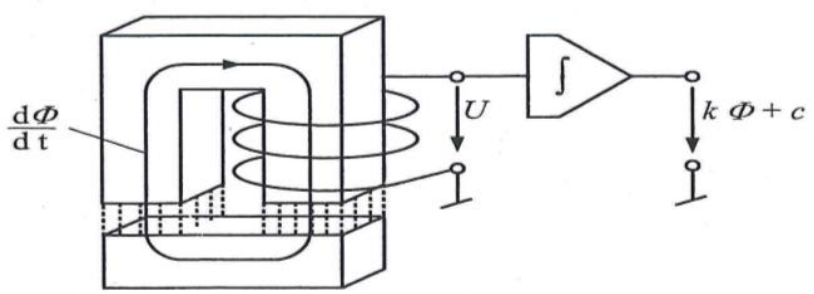

Fig. 9. Flux measurement with coil and integrator

\section{Current Measurement With A Hall Sensor}

One of the most common method of measuring current with isolation uses a Hall sensor in a magnetic loop that is excited by one or several turns of the current $i$ which is to be measured. The flux density which is measured by the Hall sensor is equilibrated with a controller, a power amplifier and an auxiliary coil as indicated in Fig. 10. The zero balance situation is reached when the flux from the current presence in the auxiliary winding with the number of $\mathrm{n}$ turns is opposite to the flux that is generated by the current $i$ to be measured. The input signal $U_{i}$ of the power amplifier is a direct measure of the current $i$

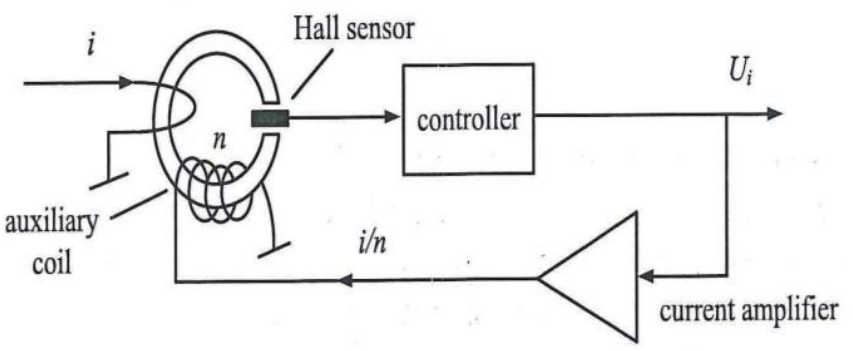

Fig. 10. Current measurement with a Hall sensor 


\section{Rotor Control Positioning}

Having a magnetic bearing which consist of an active circuit, it is very possible to control the position of the rotating rotor mass as shown in Fig. 11.

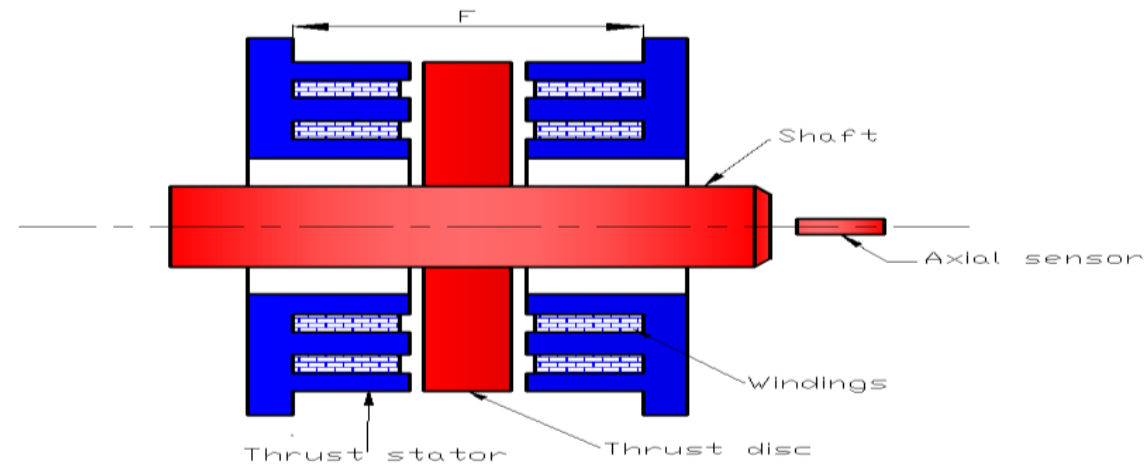

Fig. 11. Position of a rotating rotor

The rotor control position is typically based on a position sensor which has a very important function since in the setup flywheel energy storage system the system requires adequate control system not only controlling the AMB circuits but also the electrical machine such as the industrial flywheel machinery.

\section{Conclusion}

Magnetic flux density in active magnetic bearings have been discussed and presented in this paper. The flux path shows that bias flux flows into the rear side of the stator pole more than it flows into the upper portion of the rotor as presented in Fig. 3 .

In capacitive measuring method, the sensor and the opposing object which is to be measured forms one electrode of the plate, as such during the measuring process an alternating current which consists of a constant frequency is made to run through the sensor in which the sensor voltage is proportional to the clearance between the sensor electrode and the object which is to be measured which is demodulated and amplifier by a special circuit.

It could be noted that all of these areas are active foci of current AMB research. In sensing, there is a continual interest in better integration of sensing into the overall AMB structure, in reducing cost, and in reducing noise coupling between the magnet coils and the sensing head. In high precision application like grinding, milling or high speed rotating machinery such as flywheel rotors, premium is always placed on sensor performance.

An important part in the performance of the active magnetic bearing designs depends on the characteristics of the type of displacement sensor that are used. In order to measure the position of a moving rotor, contact-free sensor must be used, and moreover must be able to measure on the rotor rotating surface. In controlling magnetic flux in $\mathrm{AMB}_{\mathrm{s}}$, the geometry of the rotor, that is its surface quality as well as the homogeneity of the material of the sensor have a direct influence on the outcome of the measuring results of the system being measured.

It addition a bad surface will thus produce noise and geometry errors may cause disturbances from the rotational frequency.

\section{Acknowledgement}

Authors are grateful to the Natural Science Foundation of China (NO.51275372), Wuhan High-Tech Development Project Foundation (NO.201110921299) and The Fundamental Research Funds for the Central Universities (Wuhan University of Technology No.2012-IV-036) for supporting this research work.

\section{References}

[1]. A.V. Filator, and E.H. Maslen, Passive Magnetic Bearing for Flywheel Energy System, 7th International Conference on Vibration and control systems, 56(8), 2000, 143-148.

[2]. R. Larsonneur, G. Schweitzer, and E. Maslen, Control of the Rigid Rotor in AMBs". 10 ${ }^{\text {th }}$ Symp. on Magnetic Bearings - Theory, Design, and Application to Rotating Machinery 15(16), 2009, 170-228.

[3]. G. Schweitzer. Active Magnetic Bearings-Chances and Limitation, Sixth International conference on rotor Dynamics and Control Applications 15(17), 2002, 1-14.

[4]. G. Schweitzer, H. Bleuler. A Traxle. Basic Design and Theory of Magnetic Bearings, International conference on Active Magnetic Bearings Basics Properties and Applications, 12(6),1994,1234-1243.

[5]. P. Samantha, H. Hirani, Magnetic Bearing Configuration, Theoretical and Experimental Studies, In proceedings of the Tenth International Symposium on Magnetic Bearings, 18(16), 2008, 150-157. 
[6]. R. Larsonneur, G. Schweitzer, and E. Maslen, $6^{\text {th }}$ International conference on Magnetic Bearings - Theory, Design, and Application to Rotating Machinery. 7(8), 2009, 456-462.

[7]. M. Spirig, J. Schmied, U. Kanne. The $3^{\text {rd }}$ Symp. on Practical Examples of Magnetic Bearing Control Design using modern tools. ASME Journal of Engineering for Gas Turbine and Power Controls, 17(18), 2002, 1025-1031.

[8]. R. Larsonneur, R. Stewart, and A. Traxler, A. Active Magnetic Bearing Control of Magnetic Bearing Systems with Imbalance. In Proceedings of the 5th International Symposium on Magnetic Bearings, 8 (9), 2008, 344-354.

[9]. K. Zhang, H. Zhao. Research on Flywheel Suspended by AMB, s with Significant Gyroscopic Effect, Journal of Chinese Mechanical Engineering, 17(1), 2004, 34-39.

[10]. E. Knopf, R. Nordmann. Active Magnetic Bearings for the Identification of Dynamic Characteristics of Fluid Bearings, In 6th International Symposium on Magnetic Bearings, 14(10), 1998, 34-36. 